\title{
Multiplex immunofluorescence to measure dynamic changes in tumor-infiltrating lymphocytes and PD-L1 in early-stage breast cancer
}

Katherine Sanchez ${ }^{1,2}$, Isaac Kim ${ }^{1,2}$, Brie Chun ${ }^{1,2}$, Joanna Pucilowska ${ }^{1,2}$, William L. Redmond ${ }^{1,2}$, Walter J. Urba ${ }^{1,2}$, Maritza Martel ${ }^{3}$, Yaping Wu ${ }^{1,2}$, Mary Campbell ${ }^{3}$, Zhaoyu Sun ${ }^{1}$, Gary Grunkemeier ${ }^{4}$, Shu Ching Chang ${ }^{4}$,

Brady Bernard ${ }^{1,2}$ and David B. Page ${ }^{1,2^{*}}$ (1)

\begin{abstract}
Background: The H\&E stromal tumor-infiltrating lymphocyte (STIL) score and programmed death ligand 1 (PD-L1) SP142 immunohistochemistry assay are prognostic and predictive in early-stage breast cancer, but are operatordependent and may have insufficient precision to characterize dynamic changes in STILs/PD-L1 in the context of clinical research. We illustrate how multiplex immunofluorescence (mIF) combined with statistical modeling can be used to precisely estimate dynamic changes in STIL score, PD-L1 expression, and other immune variables from a single paraffin-embedded slide, thus enabling comprehensive characterization of activity of novel immunotherapy agents.
\end{abstract}

Methods: Serial tissue was obtained from a recent clinical trial evaluating loco-regional cytokine delivery as a strategy to promote immune cell infiltration and activation in breast tumors. Pre-treatment biopsies and posttreatment tumor resections were analyzed by mIF (PerkinElmer Vectra) using an antibody panel that characterized tumor cells (cytokeratin-positive), immune cells (CD3, CD8, CD163, FoxP3), and PD-L1 expression. mIF estimates of STIL score and PD-L1 expression were compared to the H\&E/SP142 clinical assays. Hierarchical linear modeling was utilized to compare pre- and post-treatment immune cell expression, account for correlation of time-dependent measurement, variation across high-powered magnification views within each subject, and variation between subjects. Simulation methods (Monte Carlo, bootstrapping) were used to evaluate the impact of model and tissue sample size on statistical power.

(Continued on next page)

\footnotetext{
* Correspondence: david.page2@providence.org

'Earle A. Chiles Research Institute, 4805 N.E. Glisan St., North Tower, Suite

2N87, Portland, OR 97213, USA

2Providence Cancer Institute, Portland, OR, USA

Full list of author information is available at the end of the article
}

C C The Author(s). 2021 Open Access This article is licensed under a Creative Commons Attribution 4.0 International License, which permits use, sharing, adaptation, distribution and reproduction in any medium or format, as long as you give appropriate credit to the original author(s) and the source, provide a link to the Creative Commons licence, and indicate if changes were made. The images or other third party material in this article are included in the article's Creative Commons licence, unless indicated otherwise in a credit line to the material. If material is not included in the article's Creative Commons licence and your intended use is not permitted by statutory regulation or exceeds the permitted use, you will need to obtain permission directly from the copyright holder. To view a copy of this licence, visit http://creativecommons.org/licenses/by/4.0/. The Creative Commons Public Domain Dedication waiver (http://creativecommons.org/publicdomain/zero/1.0/) applies to the data made available in this article, unless otherwise stated in a credit line to the data. 
(Continued from previous page)

Results: mIF estimates of STIL and PD-L1 expression were strongly correlated with their respective clinical assays $(p<.001)$. Hierarchical linear modeling resulted in more precise estimates of treatment-related increases in sTIL, PD$L 1$, and other metrics such as CD8+ tumor nest infiltration. Statistical precision was dependent on adequate tissue sampling, with at least 15 high-powered fields recommended per specimen. Compared to conventional t-testing of means, hierarchical linear modeling was associated with substantial reductions in enrollment size required $(n=$ $25 \rightarrow n=13$ ) to detect the observed increases in STIL/PD-L1.

Conclusion: mIF is useful for quantifying treatment-related dynamic changes in sTILS/PD-L1 and is concordant with clinical assays, but with greater precision. Hierarchical linear modeling can mitigate the effects of intratumoral heterogeneity on immune cell count estimations, allowing for more efficient detection of treatment-related pharmocodynamic effects in the context of clinical trials.

Trial registration: NCT02950259.

Keywords: Immunotherapy, IRX-2, Multiplex immunofluorescence, Early-stage breast cancer, PD-L1, sTIL, CD8

\section{Background}

Immunotherapy with anti-programmed cell death ligand 1 (anti-PD-L1, atezolizumab) was recently approved by the Food and Drug Administration (FDA) for the indication of PD-L1-positive metastatic triple negative breast cancer (TNBC) [1, 2]. However, novel combination immuno-oncology (I-O) therapies will be required to improve efficacy in other therapeutic settings, such as for PD-L1-negative disease, or for less immunogenic breast cancer subtypes such as luminal-type hormone receptor-positive cancers. In an era when numerous I-O agents are being developed clinically [3-5], one promising avenue to accelerate drug development is to develop biomarkers to characterize immune cell (IC) and tumor cell (TC) infiltrates, enabling a comparison of pharmacodynamic effects of various I-O strategies. Here, we describe a methodology that employs multiplex immunofluorescence (mIF) in conjunction with statistical modeling to characterize IC infiltration and PD-L1 expression in the context of early-stage breast cancer (ESBC) I-O clinical trials.

The mIF assay is of particular interest in breast cancer because it may serve to complement two clinically developed I-O biomarkers, PD-L1 expression (by the Ventana SP142 assay), and the hematoxylin and eosin (H\&E) stromal tumor-infiltrating lymphocyte (sTIL) score. The SP142 PD-L1 assay is FDA-approved to identify patients with PD-L $1^{+}$TNBCs who could potentially benefit from the addition of atezolizumab to nab-paclitaxel $[1,6]$. The SP142 assay categorizes tumors as $\mathrm{PD}-\mathrm{L}^{+}$if at least $1 \%$ of the tumor area is occupied by PD- $1^{+}$immune cells (ICs). PD-L1 expression is thought to be dynamic, with biological conditions and/or therapeutic interventions potentially modifying the extent of PD-L1. While the binary designation of PD-L1 status is clinically useful, its ability to serve as a pharmacodynamic biomarker to assess for dynamic PD-L1 change is limited by its semiquantitative nature and operator dependency. Likewise, the H\&E sTIL score uses pathologist estimation of proportion of stromal area occupied by TILs on a single $\mathrm{H} \& \mathrm{E}$ slide as a general gauge of tumor immunogenicity [7]. Similar to PD-L1 testing, sTIL may be clinically useful (as it correlates with survival, chemotherapy response, and potentially immunotherapy response) [8-12]; however, several barriers limit its use as a pharmacodynamic biomarker, including suboptimal interobserver concordance related to underlying intratumoral heterogeneity of sTILs [13]. Both PD-L1 and sTILs have the limitation of being semi-quantitative assays and require a pathologist to visually estimate ICs, which may sometimes be present in low abundance.

mIF enables estimation of IC counts in high resolution across numerous high-powered magnification fields (hereafter called regions of interest, ROI) and therefore has the potential to produce more accurate and precise estimates of sTILs and PD-L1 expression, relative to the clinical assays. Furthermore, mIF permits more detailed characterization of $\mathrm{IC} / \mathrm{TC}$ interactions via single-cell quantification of numerous phenotypic surface markers, and spatial localization of cells into various tissue compartments (i.e., tumor versus stroma). Here we use a 6-marker panel of CK, CD3, CD8, FoxP3, CD163, and PD-L1 to visualize, quantify, and phenotype ICs and TCs in ESBC specimens. IC densities and PD-L1 expression are repeatedly sampled across multiple ROIs on a single slide, providing the opportunity to characterize spatial heterogeneity. With appropriate statistical modeling, the repeated sampling of ROIs can be used to improve both accuracy and precision of IC density and PD-L1 expression estimates.

Here, we report mIF data from a phase Ib study of IRX-2, a loco-regional cytokine therapy in early-stage breast cancer (ESBC) [14]. IRX-2 contains various cytokines (including interleukin (IL)-2, IL-1 $\beta$, interferon- $\gamma$, tumor necrosis factor- $\alpha$, among others) delivered subcutaneously in the distribution of regional lymphatics, 
and was previously shown to increase tumor lymphocyte infiltration in pre-operative head and neck carcinomas [15-17]. In the phase Ib breast cancer trial, IRX-2 was injected in the peri-areolar tissue (modeled after sentinel lymph node mapping) and was found to be well tolerated, achieving the primary safety endpoint as well as showing evidence of enhanced IC infiltration and lymphocyte activation (measured by RNA sequencing) [14]. Using the paired biopsy and surgical excision specimens from this trial, our objectives of this project were (1) to propose a method for harmonizing mIF with the PD-L1 SP142 and H\&E sTIL clinical assays; (2) to illustrate how hierarchical linear modeling can enhance statistical precision of IC density/PD-L1 expression estimates; and (3) to evaluate the influence of ROI sample size on overall statistical power to detect changes in ICs/ PD-L1 in the context of a clinical trial.

\section{Methods}

\section{Parent clinical trial and sample acquisition}

Samples were obtained from a pre-surgical phase Ib combination immunotherapy clinical trial (NCT02950259) [14]. The trial is completed, and detailed results have been published, with demographic information summarized in Table 1 [14]. Briefly, patients with ESBC (stage I-III) planned for definitive surgical resection (either lumpectomy or mastectomy) were considered for enrollment at the Providence Cancer Institute (Portland, OR) from May 2016 to May 2018 $(n=16)$. Inclusion criteria comprised any breast cancer subtype, resectable primary tumor $>0.5 \mathrm{~cm}$, Karnofsky Performance Status of $\geq 70 \%$, adequate organ function, absence of steroid-dependent medical conditions, and absence of prior immunotherapy. Diagnostic core biopsies and excisional tumor specimens were collected, processed, and fixed in paraffin (FFPE) per standard-ofcare clinical pathology procedures. In this study, a cytokine-based combination immunotherapy approach (IRX-2) was evaluated for feasibility, safety, and immunomodulatory activity (including flow cytometry analysis of lymphocyte subsets, $\mathrm{T}$ cell repertoire analysis, and assessment of IC infiltration). Treatment included a combination of single, low-dose cyclophosphamide (300 mg/ $\left.\mathrm{m}^{2} \mathrm{IV}\right)$ to stimulate antigen presentation and deplete $\mathrm{T}$ regulatory cells, daily oral indomethacin $(25 \mathrm{mg}$ three times daily, days 1-21) to modulate myeloid-derived suppressor cells (MDSCs), and locoregional therapy with the investigational agent, IRX-2 (2 mL subcutaneous daily $\times 10)$. IRX-2 is a physiologically derived cytokine mixture obtained by ex vivo stimulation (using phytohemagluttinin) of pooled donor peripheral blood leukocytes, from which a stable cytokine mixture is obtained under GMP conditions [18]. The cytokine mixture was delivered subcutaneously adjacent the areola using a method that recapitulated axillary sentinel lymph node mapping, potentially allowing for transmission of cytokines to the tumor-draining lymph node, the putative site of antigen presentation. The study protocol was approved by the Providence Portland Medical Center IRB committee and was conducted in accordance with the ethical standards established by the Declaration of Helsinki.

\section{Stromal TIL scoring by routine hematoxylin and eosin (H\&E)}

For each treated subject, $5 \mu \mathrm{m}$ FFPE tissue sections were cut from the core and excision specimens. H\&E staining was performed and reviewed by a breast pathologist to confirm the presence of tumor and to evaluate fixation quality. Tissue samples stained by conventional H\&E were digitally scanned with Leica SCN400F platform at 20X and maginfication $220 \mathrm{x}-400 \mathrm{x}$ to facilitate blinded evaluation of sTILs using guidelines published by the International Immuno-Oncology Biomarker Working Group on Breast Cancer (i.e., sTILs working group) [7]. The average of sTIL scores of two blinded pathologists were reported as the percentage of stromal area occupied by lymphocytes within areas of invasive carcinoma, excluding areas of carcinoma in situ, necrosis, normal breast/adipose tissue, and biopsy trauma [7, 19].

\section{PD-L1 positivity by Ventana SP142 assay}

Additional 5- $\mu \mathrm{m}$ FFPE slides were stained for clinical PD-L1 scoring using the SP142 assay (Ventana Medical Systems Inc., Tucson, AZ, USA). ICs were scored by two blinded pathologists using published guidelines [6, 20], reported as the proportion of tumor area occupied by PD-L1-staining ICs of any intensity. PD-L1 was scored using the recommended standardized cutoffs (IC0- $<1 \%$, IC1- $\geq 1 \%$ to $<5 \%, \mathrm{IC} 2 \geq 5 \%$ to $10 \%$, and IC3 $\geq 10 \%$ ) [6]. In metastatic TNBC, anti-PD-L1 therapy (atezolizumab) is approved in combination with nab-paclitaxel for the treatment of PD-L1 tumors, which corresponds with scores of IC1-IC3 [1, 2]. To serve as internal control for sTIL and PD-L1 score, a contemporary cohort of untreated stage I-III biopsy and matched surgical resection samples $(n=14)$ were analyzed for PD-L1 and sTIL score.

\section{Multiplex immunofluorescence staining and image acquisition}

Additional 5- $\mu \mathrm{m}$ FFPE slides were stained for mIF. Staining methods were validated by the EACRI IHC Core at the Providence Cancer Institute (Portland, OR) and are previously reported [21]. Briefly, sections were deparaffinized and subjected to heat-induced epitope retrieval in Tris-EDTA buffer (pH 9.0). 6-plex panel mIF was performed using the following antibodies: anti- 
Table 1 Sample summary and clinical results

\begin{tabular}{|c|c|c|c|c|c|c|c|c|c|c|c|c|c|c|c|}
\hline \multirow[b]{2}{*}{ ID } & \multirow[b]{2}{*}{ TNM } & \multirow[b]{2}{*}{ Ki67 } & \multirow[b]{2}{*}{ Grade } & \multirow[b]{2}{*}{ ER/PR } & \multirow{2}{*}{ HER2 } & \multicolumn{4}{|c|}{ Pre-treatment } & \multicolumn{4}{|c|}{ Post-treatment } & \multicolumn{2}{|c|}{ Fold change } \\
\hline & & & & & & $\begin{array}{l}\text { STIL } \\
\text { H\&E } \\
(\%)\end{array}$ & $\begin{array}{l}\text { STIL mIF* } \\
\text { (count// } \\
\text { pixel) }\end{array}$ & $\begin{array}{l}\text { PD-L1 mIF* } \\
\text { (count/ } \\
\text { pixel) }\end{array}$ & $\begin{array}{l}\text { PD-L1 } \\
\text { SP142 }\end{array}$ & $\begin{array}{l}\text { STIL } \\
\text { H\&E } \\
(\%)\end{array}$ & $\begin{array}{l}\text { sTIL mIF* } \\
\text { (count/ } \\
\text { pixel) }\end{array}$ & $\begin{array}{l}\text { PD-L1 mlF* } \\
\text { (count/ } \\
\text { pixel) }\end{array}$ & $\begin{array}{l}\text { PD-L1 } \\
\text { SP142 }\end{array}$ & $\begin{array}{l}\text { STIL } \\
\text { H\&E }\end{array}$ & $\begin{array}{l}\text { PD-L1 } \\
\text { SP142 }\end{array}$ \\
\hline 1 & $\begin{array}{l}\text { pT1c, } \\
\text { N1 }\end{array}$ & $11 \%$ & 2 & $\begin{array}{l}+/+ \\
(100 \% / \\
100 \%)\end{array}$ & - & 8.75 & 0.48 & 0.07 & ICO & 12.5 & 0.59 & 0.13 & ICO & +0.4 & 0 \\
\hline 2 & $\begin{array}{l}\text { pT2c, } \\
\text { pN2 }\end{array}$ & $19 \%$ & 2 & $\begin{array}{l}+/+ \\
(96 \% / \\
90 \%)\end{array}$ & - & 13.75 & 1.35 & 1.04 & ICO & 20 & 1.53 & 0.87 & $\mathrm{n} / \mathrm{a}$ & +0.5 & $n / a$ \\
\hline 3 & $\begin{array}{l}\text { pT2, } \\
\text { N1 }\end{array}$ & $17 \%$ & 2 & $\begin{array}{l}+/+ \\
(98 \% / \\
100 \%)\end{array}$ & - & 1.75 & 0.13 & 0.07 & ICO & 1.75 & 1.25 & 0.35 & $\mathrm{IC} 1$ & 0 & +1 \\
\hline 4 & pT2N1 & $75 \%$ & 3 & $\begin{array}{l}-/- \\
(0 \% / 0 \%)\end{array}$ & + & 6.25 & 0.23 & 0.24 & $\mathrm{IC} 1$ & 10 & 0.74 & 0.68 & IC2 & +0.6 & +1 \\
\hline 5 & $\begin{array}{l}\text { pT1c, } \\
\text { N1 }\end{array}$ & $7 \%$ & 1 & $\begin{array}{l}+/+ \\
(98 \% / \\
92 \%)\end{array}$ & - & 3.75 & 0.88 & 0.18 & ICO & 5 & 0.34 & 0.09 & $\mathrm{IC} 1$ & +0.3 & +1 \\
\hline 6 & $\mathrm{~T} 2, \mathrm{~N} 1$ & $73 \%$ & 3 & $\begin{array}{l}-/- \\
(0 \% / 0 \%)\end{array}$ & + & 18.75 & 0.84 & 0.01 & ICO & 18.75 & 0.94 & 0.40 & ICO & 0 & 0 \\
\hline 7 & $\mathrm{~T} 2, \mathrm{~N} 1$ & $55 \%$ & 3 & $\begin{array}{l}+/+ \\
(98 \% / \\
100 \%)\end{array}$ & - & 18.75 & 1.18 & 0.54 & $\mathrm{IC} 1$ & 20 & 1.24 & 0.64 & $\mathrm{n} / \mathrm{a}$ & +0.1 & $\mathrm{n} / \mathrm{a}$ \\
\hline 8 & $\begin{array}{l}\text { pT2, } \\
\text { No }\end{array}$ & $50 \%$ & 3 & $\begin{array}{l}+/+ \\
(100 \% / \\
42 \%)\end{array}$ & - & 7.5 & 1.28 & 0.97 & $\mathrm{IC} 1$ & 16.25 & 1.68 & 1.30 & IC1 & +1.2 & 0 \\
\hline 9 & $\begin{array}{l}\text { pT1b, } \\
\text { pNo }\end{array}$ & $11 \%$ & 2 & $\begin{array}{l}+/+ \\
(100 \% / \\
100 \%)\end{array}$ & - & 1 & 0.06 & 0.06 & ICO & 5 & 0.27 & 0.23 & ICO & +4 & 0 \\
\hline 10 & $\begin{array}{l}\text { T1c, } \\
\text { No }\end{array}$ & $33 \%$ & 2 & $\begin{array}{l}+/+ \\
(100 \% / \\
99 \%)\end{array}$ & - & 11.5 & 0.41 & 0.38 & $\mathrm{ICl}$ & 18.75 & 1.48 & 1.33 & IC3 & +0.6 & +2 \\
\hline 11 & $\begin{array}{l}\text { pT1c, } \\
\text { pN1a }\end{array}$ & $38 \%$ & 3 & $\begin{array}{l}+/- \\
(69 \% / \\
0 \%)\end{array}$ & + & 6.25 & 0.35 & 0.22 & $\mathrm{ICl}$ & 5 & 1.59 & 0.65 & IC2 & -0.2 & +1 \\
\hline 12 & $\begin{array}{l}\text { pT2, } \\
\text { No }\end{array}$ & $12 \%$ & 3 & $\begin{array}{l}+/+ \\
(98 \% / \\
100 \%)\end{array}$ & + & 61.25 & 2.21 & 1.81 & $\mathrm{IC} 1$ & 72.5 & 3.52 & 1.99 & IC3 & +0.2 & +2 \\
\hline 13 & $\begin{array}{l}\text { pT2, } \\
\text { pNO }\end{array}$ & $87 \%$ & 3 & $\begin{array}{l}+/+ \\
(100 \% / \\
7 \%)\end{array}$ & - & 31.25 & 1.69 & 0.06 & $\mathrm{ICl}$ & 55 & 1.77 & 0.98 & IC3 & +0.8 & +2 \\
\hline 14 & N/A & N/A & 1 & $\begin{array}{l}+/+ \\
(100 \% / \\
100 \%)\end{array}$ & - & 1 & $\mathrm{n} / \mathrm{a}$ & $\mathrm{n} / \mathrm{a}$ & n/a & 1 & $\mathrm{n} / \mathrm{a}$ & $\mathrm{n} / \mathrm{a}$ & $\mathrm{n} / \mathrm{a}$ & 0 & $\mathrm{n} / \mathrm{a}$ \\
\hline 15 & $\begin{array}{l}\text { pT1c, } \\
\text { NO }\end{array}$ & $30 \%$ & 2 & $\begin{array}{l}+/+ \\
(100 \% / \\
50 \%)\end{array}$ & - & 3.5 & 1.26 & $\mathrm{n} / \mathrm{a}$ & ICO & 7 & 1.14 & 0.76 & IC2 & +1 & +2 \\
\hline 16 & $\begin{array}{l}\text { pT1b, } \\
\text { N0 }\end{array}$ & $95 \%$ & 3 & $\begin{array}{l}-/- \\
(0 \% / 0 \%)\end{array}$ & - & 1 & 0.38 & 0.03 & ICO & 13.75 & 0.99 & 0.06 & IC3 & $\begin{array}{l}+ \\
12.8\end{array}$ & +3 \\
\hline
\end{tabular}

TNM stage by the TNM Classification of Malignant Tumors, Ki67\% of Ki67-positive tumor cells, ER estrogen receptor, $P R$ progesterone receptor, $H E R 2$ human epidermal growth factor receptor 2, sTIL stromal tumor-infiltrating lymphocyte, H\&E hematoxylin and eosin stain, $P D-L 1$ programmed death ligand 1 , SP142 Ventana PD-L1 assay, IC immune cell; ${ }^{*} \times 10^{-3}$

FoxP3 (clone 236A/E7, dilution 1:400, Abcam), anti-PDL1 (clone E1L3N, dilution 1:1600, Cell Signaling), antiCD8 (clone SP16, dilution 1:400, Spring Bioscience), anti-CD3 (clone SP7, dilution 1:600, Spring Bioscience), anti-CD163 (clone MRQ26, dilution 1:4, Ventana), anti-
CK (clone AE1/AE3, dilution 1:3000, DAKO). Alternative PD-L1 clones are available and are not evaluated here in the context of mIF (SP263, SP142, and 22C3); however, the E1L3N clone was demonstrated to have comparable staining results to these antibodies in a 
recent clinical validation study [22]. Anti-mouse or rabbit HRP (Biocare Medical) was used as the primary antibody. TSA-conjugated fluorophores (PerkinElmer) were used to visualize each biomarker: Opal 690 (PDL1), Opal 650 (CD8), Opal 620 (CD163), Opal 570 (CK), Opal 540 (CD3), and Opal 520 (FoxP3). Three percent $\mathrm{H}_{2} \mathrm{O}_{2}$ and microwave treatment in citrate buffer $\mathrm{pH} 6.0$ was performed to prevent cross-reactivity. Tissue slides were incubated with DAPI as counterstain and coverslipped with Prolong antifade mountant (Thermo Fisher Scientific). Whole slides were scanned and digitized at $x$ 10 magnification (PerkinElmer Vectra 3.0) for gross visualization of the tumor, with non-overlapping regions of interest (ROIs) scanned at $\times 20\left(0.36 \mathrm{~mm}^{2}\right)$ for quantification. ROIs were selected by a study pathologist and included all available tumor-bearing areas containing at least some ICs (>100 mononuclear cells or more). Regions with empty spaces due to large vasculature, cutting artifacts, or other artifacts were avoided. The number of ROIs per slide ranged from 8 to 32 (mean 16). The workflow is graphically depicted in Fig. 1, and additional details on method are furnished upon request.

\section{Data analysis}

InForm software (PerkinElmer, package 2.4) was used to segment tumor regions (stroma versus tumor) and phenotype cells based upon marker expression. Technical details on the method [23], step-by-step instructions [24], and examples of application in other ESBC datasets are published [25]. Four representative ROIs for each specimen were used for training and capturing the heterogeneity of staining. The process can be summarized in four steps. First, digital images were processed and converted to data matrices according to optical density. Second, the ROIs were segmented into tumor and stromal compartments, which requires a manual step of selecting several small representative areas (containing 3-15 nuclei) for each compartment (using $\mathrm{H} \& \mathrm{E}$ as a benchmark). InForm uses these selections to train and segment the remaining regions, which were then manually compared with H\&E for accuracy. Third, cells were labeled according to the most likely phenotype using an Inform-based machine-learning algorithm guided by manual selection of several cells per phenotype of interest. In this experiment, cells were categorized according to the following phenotypes: tumor cells $\left(\mathrm{CK}^{+}\right)$, helper $\mathrm{T}$ cells $\left(\mathrm{CD}^{+} \mathrm{CD}^{-}\right)$, cytotoxic $\mathrm{T}$ cells $\left(\mathrm{CD}^{+} \mathrm{CD}^{+}\right)$, regulatory $\mathrm{T}$ cells $\left(\mathrm{T}\right.$ reg, $\left.\mathrm{CD}^{+}{ }^{+} \mathrm{FoxP}^{+}\right)$, macrophages $\left(\mathrm{CD}_{163^{+}}\right)$, and other stromal cells (DAPI+ only). PD-L1 expression was analyzed as both a continuous variable (reporting mean quantitative immunofluorescence [QIF] intensity for each cell), as well as a binary PD-L1+/- phenotype (described in the "Results" section). Next, image reports and phenotype maps were generated for each ROI. Finally, output files were generated containing per-cell observations with the following features: patient identification number, sample type (pre-treatment biopsy versus post-treatment excision), ROI unique identifier, cellular phenotype, tissue compartment (tumor versus stroma) and areas for each compartment, Cartesian coordinates ( $x$ - and $y$-axes), and mean cellular PD-L1 QIF intensity (illustrated in Fig. 1).

\section{Statistical methods}

An important goal was to evaluate a statistical approach that could account for heterogeneity within different areas of the tumor, thus improving reliability of estimating overall IC count and PD-L1 expression. A Poisson generalized linear mixed model (GLMM) was used with a log-linear effect of prevalence, an offset of log (area) to make the expected number of cells proportional to the area, using the function "glmer" in R package "Ime4." This well-described model [26, 27] accounts for differences in stromal/tumor area within each ROI, correlation of time-dependent measurements, and variation among patients and ROIs when estimating the relative influence of treatment exposure on IC density or PD-L1 expression. The model was selected as the best fit by likelihood ratio test, AIC and BIC criterion based on the observed data. The formal formula of the model can be written as $\log \left(\lambda_{i j}\right)=$ offset $(\log ($ Area $))+\beta_{0}+\beta_{1} T_{i}+b_{i}+$

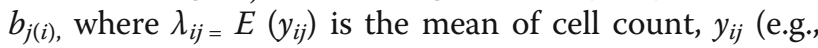
CD3 cell counts) in the $j$ th ROI of the $i$ th subject, with $T_{i}$ being a binary variable indicating post- versus pretreatment, $b_{i}$ and $b_{j(i)}$ representing random effects pertaining to $i$ th subject and $j$ th ROI. In the model, counts are adjusted for the compartment area. Treatmentassociated effects (i.e., fold change [FC] in density from pre- to post-treatment) with $95 \%$ confidence intervals were estimated by exponentiation of the coefficient for post-treatment.

\section{Results}

Evaluation of TILs by mIF

IRX-2 was previously shown to increase the mean H\&E sTIL score, upregulate PD-L1 expression, and upregulate immune-related gene signatures [14]. We sought to develop a method of measuring sTILs using mIF that would closely recapitulate the H\&E sTIL score $\left(\mathrm{sTIL}_{\mathrm{H} \& \mathrm{E}}\right)$, but potentially with greater precision compared to the semi-quantitative clinical assays. The sTILs working group guidelines define sTILs as all stromal mononuclear cells, which include lymphocytes and plasma cells but exclude other ICs such as macrophages, with the score being a percentage of stromal area occupied by these cells $[7,19]$. To recapitulate this, we defined the sTIL $L_{\mathrm{mIF}}$ score as the sum of counts of all stromal $\mathrm{T}$ cells (helper, cytotoxic, and regulatory) 


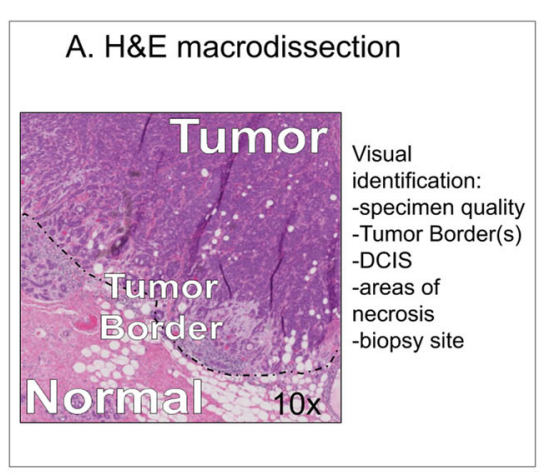

\section{B. $\mathrm{mIF}$ macrodissection}

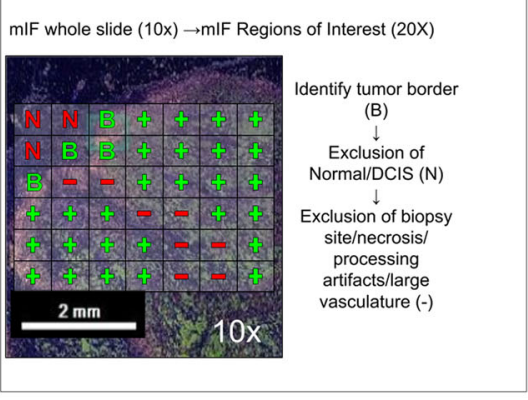

\section{C. mIF Regions of Interest (ROI)}

6 IF markers (at different wavelengths) $\rightarrow 1$ Composite mIF Image (20x)
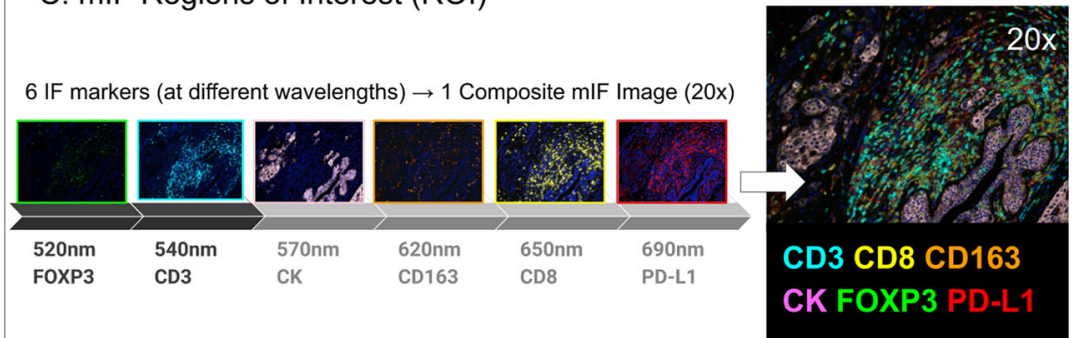

\section{Cell and tissue segmentation}

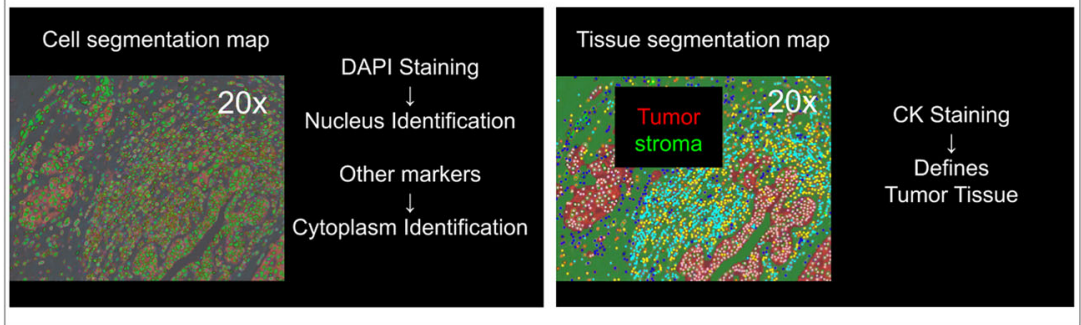

\section{E. Cellular phenotyping}

F. PD-L1 quantification per cell
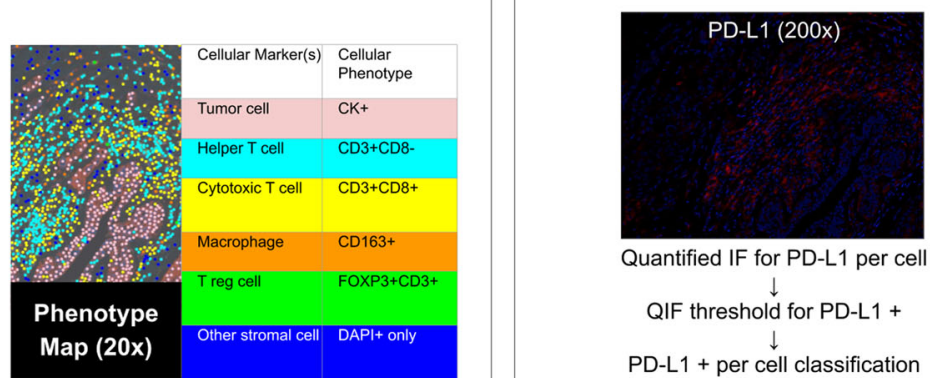

Quantified IF for PD-L1 per cell

QIF threshold for PD-L1+

$\downarrow$

PD-L1 + per cell classification

\section{G. Data Processing}

\begin{tabular}{|c|c|c|c|}
\hline Primary & $\begin{array}{l}\text { Per cell data: } \\
\text { Patient ID \# }\end{array}$ & \multirow{6}{*}{ R pipeline } & \multirow{6}{*}{$\begin{array}{c}\text { Data } \\
\text { Manipulation }\end{array}$} \\
\hline Output & Cell phenotype & & \\
\hline 1 cell = & $X, Y$ Coordinates & & \\
\hline 1 observation & Pre vs Post & & \\
\hline IRX Trial & $\begin{array}{c}\text { Intratumoral } \\
\text { stroma vs Tumor }\end{array}$ & & \\
\hline$N=985,564$ & PD-L1 QIF & & \\
\hline
\end{tabular}

Fig. 1 (See legend on next page.) 
(See figure on previous page.)

Fig. 1 Illustration of mIF workflow and staining. Refer to supplemental figure 2, 3, 4 for example mIF images, validation staining, and examples of QIF levels across phenotypes using the machine-learning based (InForm) phenotyping method. mIF: multiplex immunofluorescence; H\&E:

hematoxylin and eosin; DCIS: ductal carcinoma in situ; Bx: biopsy; CK: cytokeratin; FOXP3: forkhead box P3; PD-L1: programmed death ligand 1; ROI: region of interest; DAPI: 4',6-diamidino-2-phenylindole; T reg: T-regulatory; T-S: tumor-stromal; QIF: quantitative immunofluorescence

divided by stromal area (Table 2). Plasma cells are not captured by this panel and may be present in ESBC, but are usually clustered in sparse ROIs [28]. Results are illustrated in Table 1 and Fig. 2a, b. sTIL $\mathrm{H}_{\mathrm{HE}}$ and sample

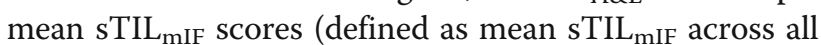
ROI) were correlated for both pre-treatment $\left(r^{2}=0.59\right.$, $p<.001)$ and excisional samples $\left(r^{2}=0.63, p<.001\right)$. sTIL $_{\mathrm{mIF}}$ scores varied substantially across ROIs within a sample, with a mean coefficient of variation (CV) of 0.61 (range 0.27-1.44), indicative of intra-sample heterogeneity.

mIF allows for evaluation of treatment-related effects on specific cell lineages across both stromal and tumor compartments. Table 3 and Fig. 3 illustrate the effects of IRX-2 on stromal and tumor cell density observed across various lineages. We evaluated therapy-related fold changes in cell count using the paired $t$-test versus adjusted counts using the hierarchical linear model. The adjusted estimates of fold change using the hierarchical linear model tended to be higher and with smaller $p$ values, relative to estimates of fold change using the paired $t$-test. IRX-2 was associated with significant increases in stromal cytotoxic $\mathrm{T}$ cells and helper $\mathrm{T}$ cells, but no change in regulatory $\mathrm{T}$ cells or macrophages. Within the tumor compartment, therapy was associated with increases in cytotoxic $\mathrm{T}$ cells; however, this only achieved significance using the hierarchical modeling approach. We also evaluated cellular ratios to evaluate for therapy-related shifts in IC phenotype predominance (supplemental table 1) and identified a reduction in the macrophage/ $\mathrm{T}$ cell ratio (median FC -0.56 , mean FC -.091 ; range -0.99 to +3 ), and the regulatory $\mathrm{T}$ cell /cytotoxic $\mathrm{T}$ cell ratio (median $\mathrm{FC}-0.78$; mean $\mathrm{FC}-0.58$; range -0.90 to +0.75$)$.

\section{Evaluation of PD-L1 expression by mIF}

IRX-2 is associated with increases in PD-L1 expression using the SP142 clinical assay $\left(\mathrm{PDL} 1_{\mathrm{SP} 142}\right)$ relative to untreated controls (Table 1). We sought to develop a method of measuring PD-L1 using mIF (PDL1 $1_{\mathrm{mIF}}$ ) that would closely recapitulate the PDL1 $1_{\mathrm{SP} 142}$ score. By SP142, pathologists visually classify individual ICs as

Table 2 Summary of metrics and definitions

\begin{tabular}{|c|c|c|c|c|}
\hline Metric & Notation & Formula & $\begin{array}{l}\text { Analysis level } \\
\text { (cell, ROI, } \\
\text { slide) }\end{array}$ & Attributes \\
\hline \multicolumn{5}{|c|}{ Count/density metrics } \\
\hline $\begin{array}{l}\text { sTIL } \\
\text { score }\end{array}$ & STIL $L_{H E}$ & $\begin{array}{l}\% \text { of stromal area occupied } \\
\text { by TILs on one H\&E slide }\end{array}$ & Slide & $\begin{array}{l}\text { Generalized metric for presence/absence of immune cells, prognostic in } \\
\text { breast cancer and predictive of chemotherapy benefit and potentially } \\
\text { immunotherapy benefit (references }[10,11] \text { ) }\end{array}$ \\
\hline $\begin{array}{l}\text { mlF sTIL } \\
\text { score }\end{array}$ & $\mathrm{sTIL} \mathrm{L}_{\mathrm{mIF}}$ & $\frac{\text { Count }_{5 \mathrm{CD} 3+/ \mathrm{sCD} 8+/ \mathrm{sFOXP3}}}{\text { area }_{5}}$ & $\mathrm{ROI}$ & $\begin{array}{l}\text { Quantification of therapy-related changes in overall TILs. Analogous to the } \\
\text { H\&E sTIL score and therefore may have potential as a prognostic/predictive } \\
\text { marker }\end{array}$ \\
\hline $\begin{array}{l}\text { Cell } \\
\text { count }\end{array}$ & Count $_{\mathrm{CD} 8}$ & 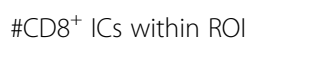 & $\mathrm{ROI}$ & \\
\hline $\begin{array}{l}\text { Cell } \\
\text { density }\end{array}$ & Density $_{\text {SCD8 }}$ & $\frac{\text { Count }_{\mathrm{sCD} 8}}{\text { Area }_{5}}$ & $\mathrm{ROI}$ & $\begin{array}{l}\text { May be a more reliable measurement of IC quantity because it adjusts for } \\
\text { differences in stroma/tumor compartmentalization across tumor regions }\end{array}$ \\
\hline \multicolumn{5}{|c|}{ PD-L1 metrics } \\
\hline $\begin{array}{l}\text { SP142 } \\
\text { PD-L1 } \\
\text { score }\end{array}$ & $\mathrm{PDL} 1_{\mathrm{SP} 142}$ & $\begin{array}{l}\% \text { of tumor area occupied } \\
\text { by PDL1-positive ICs }\end{array}$ & Slide & $\begin{array}{l}\text { Categorical metric for ascertaining IC PD-L1 positivity, predictive of im- } \\
\text { munotherapy response (anti-PD- L1) in triple negative breast cancer }\end{array}$ \\
\hline $\begin{array}{l}\text { PD-L1 } \\
\text { cell } \\
\text { intensity }\end{array}$ & $\mathrm{QIF}_{\mathrm{CD} 8}$ & PDL1 mean whole cell QIF & Cell & $\begin{array}{l}\text { Quantification of PD-L1 intensity on individual cells may be useful for evalu- } \\
\text { ating effect of therapies on dynamic PD- } L 1 \text { expression on tumor or ICs }\end{array}$ \\
\hline $\begin{array}{l}\text { mIF PD- } \\
\text { L1 score }\end{array}$ & $P D L 1_{\mathrm{mlF}}$ & $\begin{array}{l}\text { Count }{ }_{\mathrm{PDL} 1^{+}} \mathrm{KC} \\
\left(\mathrm{CD} 3^{+} \text {or } \mathrm{CD} 163^{+} \& \mathrm{QIF}>\right. \\
\text { 2.6) }\end{array}$ & $\mathrm{ROI}$ & $\begin{array}{l}\text { Quantification of overall number of PD-L1+ ICS. Analogous to the SP142 } \\
\text { PDL1 score and therefore may have potential as a predictive marker }\end{array}$ \\
\hline
\end{tabular}

$R O I$ region of interest, $\mathrm{mIF}$ multispectral immunofluorescence, sTIL stromal tumor-infiltrating lymphocyte, H\&E hematoxylin and eosin, $T I L s$ tumor-infiltrating lymphocytes; s stromal, IC immune cell, SP142 Ventana SP142 PD-L1 assay, PD-L1 programmed death ligand 1, QIF quantitative immunofluorescence 


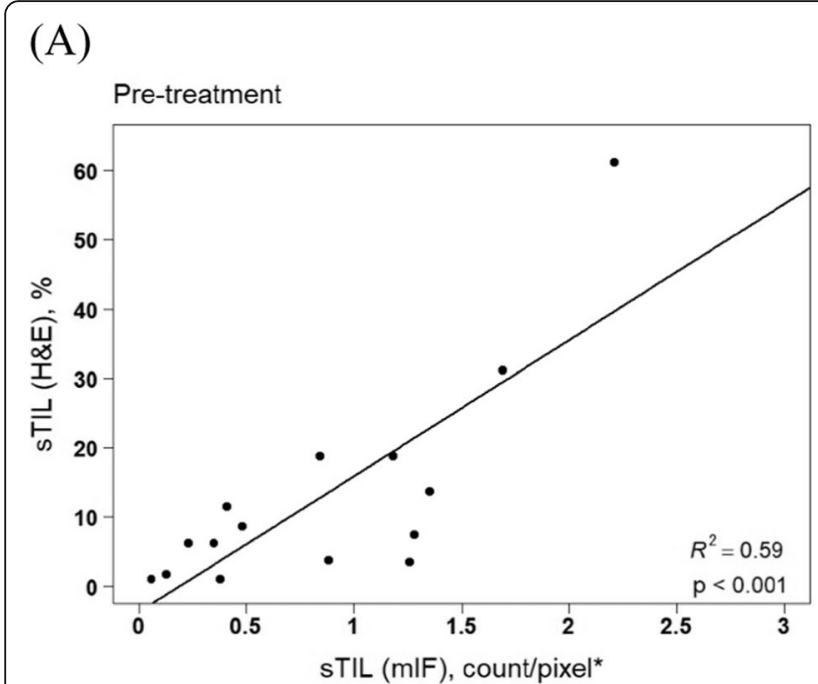

(C)

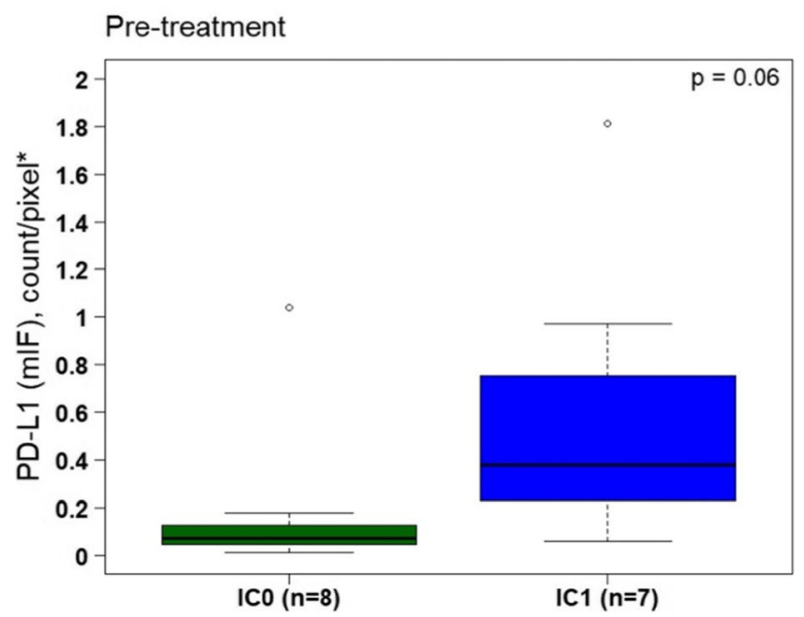

(B)

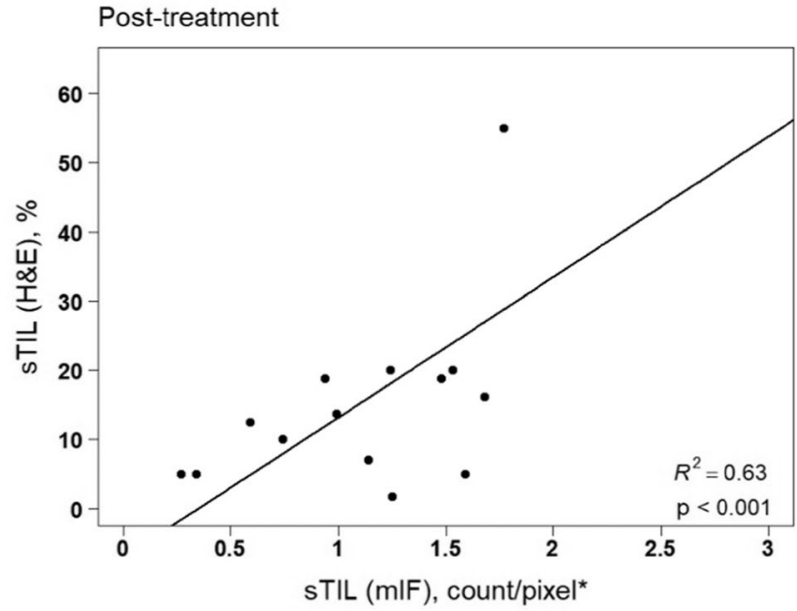

(D)

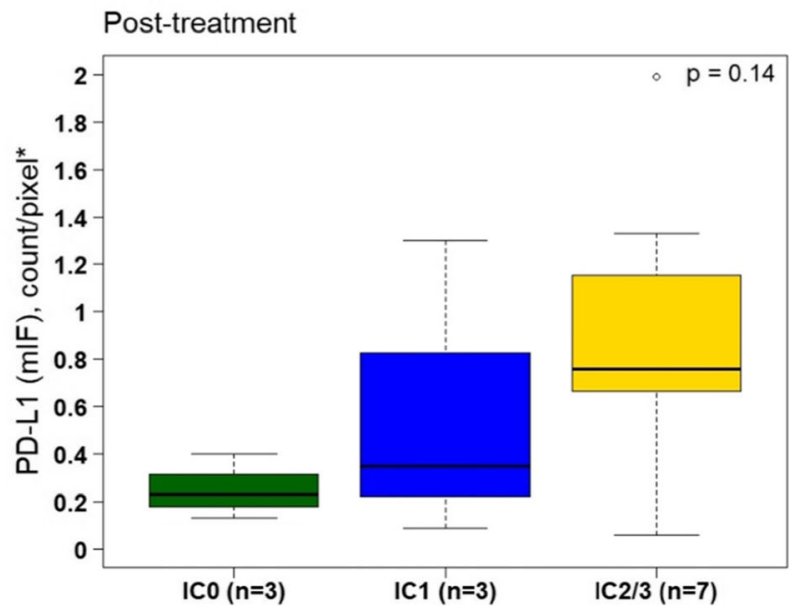

Fig. 2 Harmonization of clinical sTIL and PD-L1 assays with mIF. Correlation scatterplot of sTIL (H\&E) and sTIL (mIF) for pre-treatment (a) and posttreatment (b) with fitted linear regression lines. Mean PD-L1 mIF score comparison between PD-L1 SP142 category (IC 0, IC1, IC2/3) for pretreatment (c) and post-treatment (d) using Jonckheere-Terpstra test. sTIL: stromal tumor-infiltrating lymphocyte; mIF: multiplex immunofluorescence; IC: immune cell; PD-L1: programmed death ligand 1

PD-L1 positive versus negative, then estimate the total tumor area occupied by PD-L1-positive cells. To recapitulate this method by mIF, an accurate per-cell QIF cutoff for PD-L1 positivity would be necessary. We used InForm software to create simulated chromogenic PDL1 images derived from individual ROIs of various mIF samples. With these images, the study pathologist was asked to classify 231 randomly selected ICs of various PD-L1 QIF across 4 specimens, and with these classifications, a receiver operating characteristic (ROC) curve was generated to determine the most accurate QIF cutoff for per-cell PD-L1 positivity. The optimal QIF level was 2.6, corresponding with an AUC of 0.97 , sensitivity of $91 \%$, specificity of $99 \%$, and classification accuracy of
95\% (supplemental figure 1). Using the 2.6 cutoff, accuracy for individual specimens ranged from 92 to $100 \%$ (mean 96\%), suggesting that a QIF cutoff of 2.6 would be adequate across tumor samples. We defined the PDL $1_{\mathrm{mIF}}$ score as the count of all ICs within an ROI with QIF > 2.6. As illustrated in Table 3 and Fig. 2c, d, we demonstrate a mean 3.14-fold increase of PDL1 $1_{\mathrm{mIF}}$ IC density related to treatment. PDL $1_{\mathrm{mIF}}$ scores were correlated with the PDL $1_{\mathrm{SP} 142}$ assay, with average densities increasing concordantly according to SP142 IC category (Fig. 2c, d). In a mixed effects model that accounts for correlations across pre/post-treatment samples, PDL1 $1_{\mathrm{mIF}}$ for IC1 tumors was 2.99-fold higher $(p=.04)$ than IC0 tumors, and PDL $1_{\mathrm{mIF}}$ for $\mathrm{IC} 2 / 3$ tumors was 
Table 3 IRX-related effects on various immune cell subpopulations within stromal and tumor tissue compartments

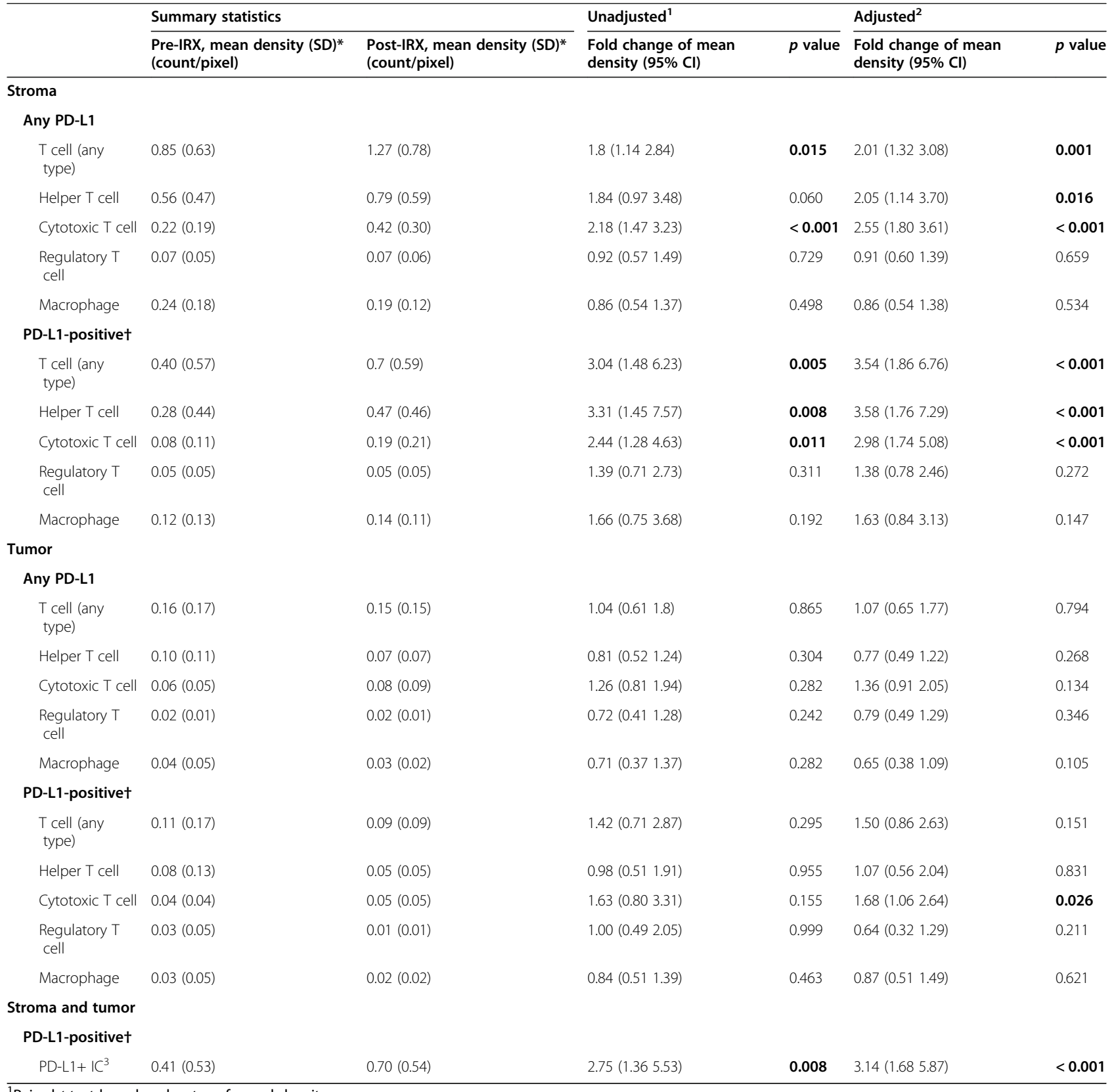

${ }^{1}$ Paired $t$-test based on log-transformed density

${ }^{2}$ Poisson Generalized Linear Mixed-Effects Model (GLMM), with a log-linear effect of prevalence, an offset of log (area) (to make the expected number of cells proportional to area), the fixed effect of time (pre vs post), and two random effects of patients and ROI, to account for the variation in patients and ROI ${ }^{*} \times 10^{-3} ;$ +PD-L1 $>2.6$

$\mathrm{T}$ cell (any type $)=$ combination of $\mathrm{CD} 3+, \mathrm{CD} 8+$, and FOXP3+ cell types; helper $\mathrm{T}$ cell $=\mathrm{CD} 3+$; cytotoxic $\mathrm{T}$ cell $=\mathrm{CD} 8+$; regulatory $\mathrm{T}$ cell $=\mathrm{FOXP3}+$; macrophage $=$ CD163

$P D-L 1$ programmed death ligand $1, C l$ confidence interval 


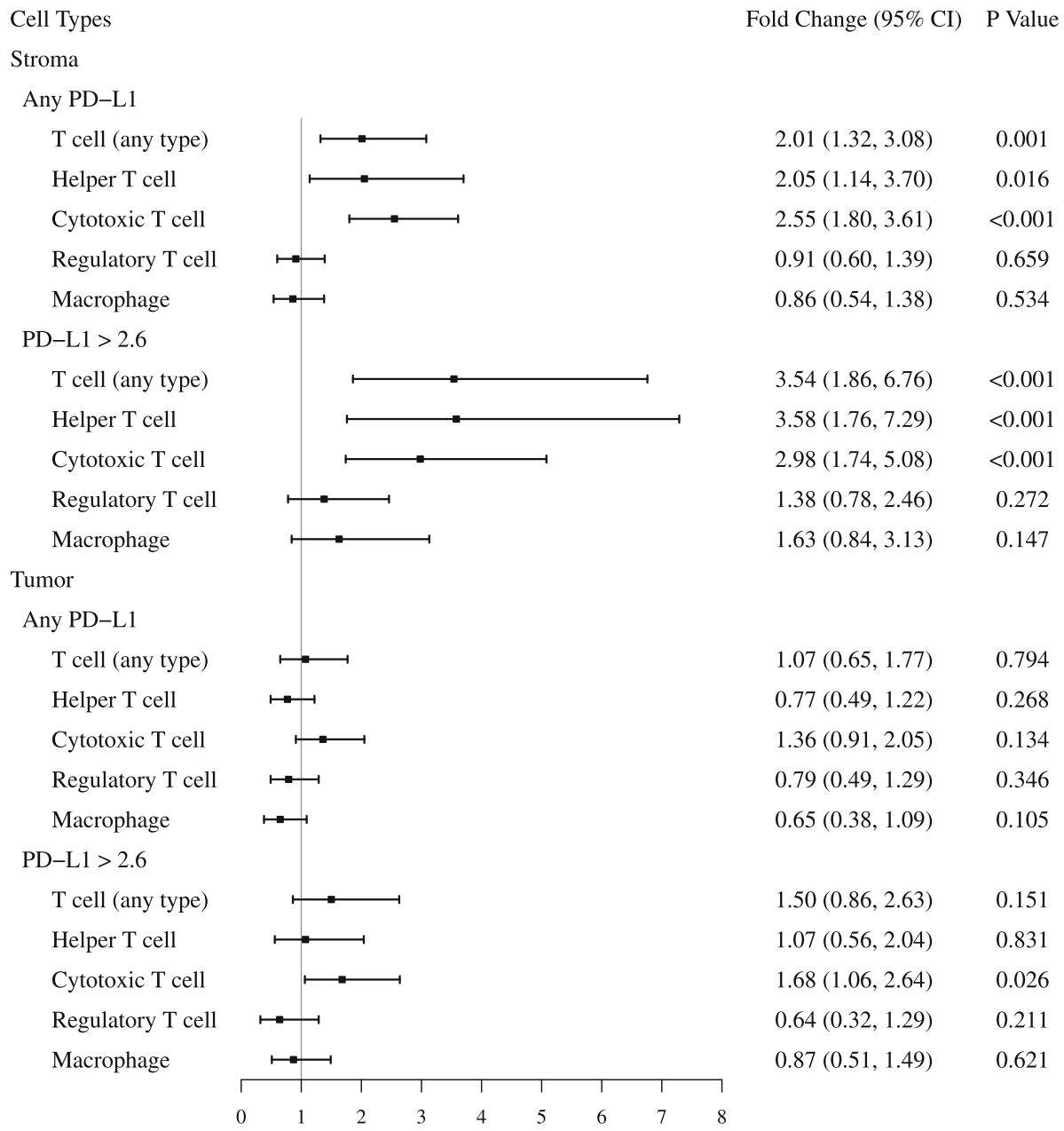

Fig. 3 Forest plot of IRX-2 immunotherapy effects on lymphocyte subsets. PD-L1: programmed death ligand 1; Cl: confidence interval

5.95-fold higher $(p=.003)$ than IC0 tumors. Similar to sTIL $_{\mathrm{mIF}}$ scores, PDL1 $1_{\mathrm{mIF}}$ scores ranged widely across ROI within samples (CV 0.78, range 0.3-1.29), highlighting the importance of adequate ROI sampling to characterize tumors.

\section{Power analyses}

Because of the computational labor associated with mIF, it is of interest to ascertain how many ROIs must be analyzed to adequately represent the entirety of the specimen. We evaluated whether fewer ROIs would be sufficient to detect treatment-related change in a clinical trial, using PD-L $1_{\mathrm{mIF}}$ and $\mathrm{sTIL}_{\mathrm{mIF}}$ scores as examples. Holding patient sample size $(n=15)$ fixed, a Monte Carlo simulation approach ( $n=1000$ simulations) was employed to calculate power across various ROI sample sizes, based on the Poisson hierarchical linear model described in section "Statistical methods", and the observed data structure [i.e., effect sizes and variations obtained post hoc from a pilot experiment] using the "simr" package in $\mathrm{R}$ [29]. The analyses show that with 15 subjects, on average, 22 ROIs within each subject would be required to detect the treatment effect ( $F C=3.14,95 \%$ $\mathrm{CI}=1.68-5.87$ ) for PD-L1 $1_{\mathrm{mIF}}$ (Fig. 4a), and 24 ROIs within each subject would be required to detect the treatment effect $(\mathrm{FC}=2.01,95 \% \mathrm{CI}=1.32-3.08)$ for sTIL $_{\mathrm{mIF}}$ (Fig. 4b), to attain at least $80 \%$ power at a significance level of 0.05 . A reduction in ROI sampling led to a substantial decrease in statistical power to detect changes in both PD-L1 $1_{\mathrm{mIF}}$ and sTIL $\mathrm{SIF}_{\mathrm{mIF}}$ (Fig. 4a, b), likely related to the high degree of variation in $\mathrm{PD}-\mathrm{L} 1_{\mathrm{mIF}}$ and sTIL $_{\mathrm{mIF}}$ across ROIs within the same specimen. In our experience, the range of evaluable ROIs per specimen was 8-32 (mean 16), and therefore in the context of similar trials, it would be advisable to evaluate as many ROIs as possible on one slide to detect effects similar to described. In studies evaluating smaller effect sizes, greater sample sizes would be required.

We compared the hierarchical linear model with more conventional methods of reporting treatment-related 


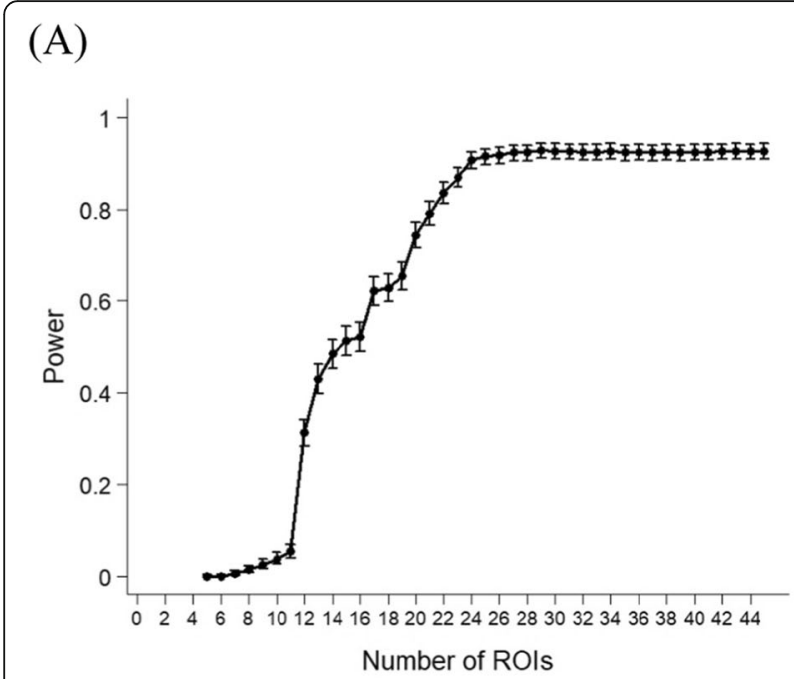

(B)

(C)
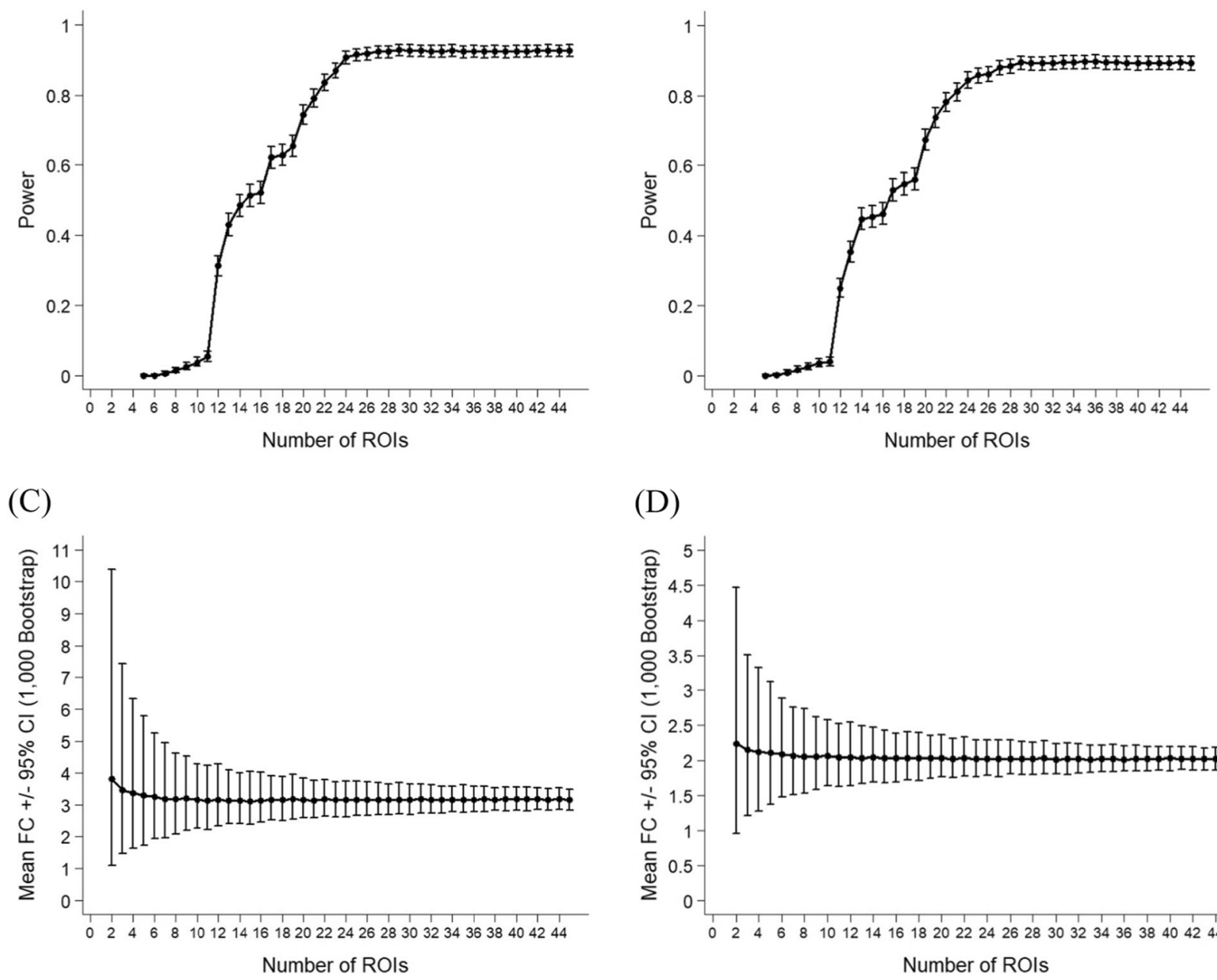

(D)

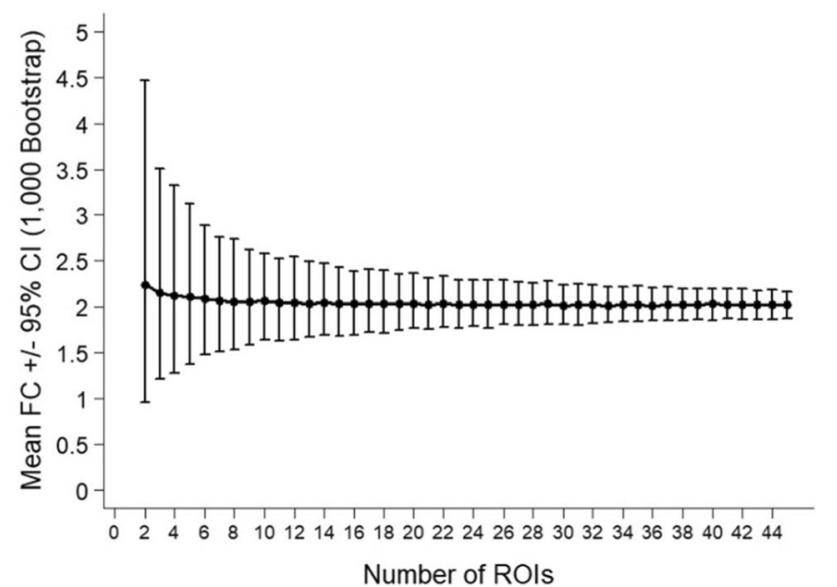

Fig. 4 Power analyses illustrating the effects of ROI sample size. a, b Power curve according to ROI size, holding patient sample size ( $n=15)$ fixed, for the PD-L1 mIF biomarker (a) and the sTIL mIF biomarker (b) produced using a Monte Carlo simulation approach ( $n=1000$ simulations), based on the generalized linear mixed effects model and the observed data structure [i.e., effect sizes and variations obtained post hoc from the pilot experiment]. c, d Mean fold change and $95 \%$ confidence intervals based upon a bootstrap simulation method (1000 simulations) according to ROI size, for the PD-L1 mIF biomarker (c) and the sTIL mIF biomarker (d). ROI: region of interest; FC: fold change; Cl: confidence interval

change in clinical trials. The most common conventional method is to test fold changes in means using the paired $t$-test. Using the above Monte Carlo method for the hierarchical linear model, holding per-specimen ROI fixed based on observed data structure, we estimated that $n=11$ patients would be required to detect a 3.14fold change in $\mathrm{PD}-\mathrm{L} 1_{\mathrm{mIF}}$, and $n=13$ patients would be required to detect a 2.01 -fold change in STIL $_{\text {mIF }}$ with $80 \%$ power at a significance level of 0.05 (supplementary table 2). However, by the paired $t$-test approach [30], a sample size of $n=13$ would be required to detect similar changes in $\mathrm{PD}-\mathrm{L} 1_{\mathrm{mIF}}(\mathrm{FC}=2.75, \mathrm{CV}=1.08)$, whereas a sample size of $n=25$ would be required to detect similar changes in sTIL $_{\mathrm{mIF}}(\mathrm{FC}=1.80, \mathrm{CV}=0.83)$. These data suggest that the hierarchical linear model is associated with increased statistical power, or reductions in required subject enrollment size, in the context of comparative trials.

Finally, we evaluated the potential impact of ROI sample size on estimation of treatment-related changes in sTILs or PD-L1 expression. Using a bootstrap method assuming a clinical trial with $n=15$ patients, fold change estimates of sTIL and PD-L1 scores were simulated 1000 times across various ROI sizes to create a mean and 95\% CI of estimated FC. As illustrated in Fig. 4c, d, ROI sample sizes of $<10$ were associated with wide CIs, whereas ROI sample sizes of $>15$ were sufficient to optimize accuracy. 


\section{Discussion}

Innumerable I-O strategies show promise in preclinical breast cancer models either as monotherapy or in combination with approved therapies ( $\mathrm{T}$ cell agonists, trastuzumab, chemotherapy, radiotherapy, or targeted therapy) $[1-4,31-33]$. Pre-operative I-O clinical trials in ESBC provide the opportunity to efficiently compare pharmacodynamic activity using serial tissue-based comparative biomarkers, while also providing pathologic outcomes as a meaningful surrogate of disease-free recurrence [34]. mIF has been proposed as a promising biomarker, as it has been shown to be concordant with clinical PD-L1 assays in ESBC [35], and in a recent meta-analysis outperformed clinical PD-L1 testing, quantification of tumor mutational burden, or gene expression profiling in predicting immunotherapy response [36]. Here, we provide additional guidance on how mIF can be used as a pharmacodynamic biomarker in the context of ESBC I-O pre-operative clinical trials. We show that mIF estimates of PD-L1 expression and sTIL/IC density correlate with the validated clinical assays, but with higher resolution to measure treatment-related pharmacodynamic changes. It has recently been suggested that both PD-L1 and sTIL clinical assays be co-analyzed to enhance predictive/prognostic performance [37]. As illustrated in this manuscript, mIF provides granular detail on single-cell PD-L1 expression across cellular phenotypes, which can be used to categorize tumors based upon ratios of PD-L1-expressing cells, phenotypic predominance patterns of PD-L1+ cells (i.e., macrophage v. lymphocyte), and spatial patterning of PD-L1. As a future direction of investigation, we propose that clinical investigators prioritize the inclusion of mIF in clinical trials in tandem with the clinical assays, so the unique predictive/prognostic utility of these added data can be adequately interrogated.

We identified several aspects of mIF that might be useful in addressing the pitfalls of clinical H\&E sTIL assessment, which were recently summarized from the RING studies [13]. First, by H\&E, it was found that nonlymphocyte cells or intraepithelial TILs could be misclassified for sTILs by pathologists. mIF could substantially mitigate this source of error, by employing multiple cell surface markers to accurately classify lymphocytes. Second, it was found that pathologists exhibited different set-points/scales for quantifying sTILs by $H \& E$, resulting in substantial inter-observer discordance. This pitfall could be in the future be mitigated by mIF once the staining, imaging, and analysis workflow becomes harmonized across institutions. Efforts are ongoing via the National Institutes of Health Cancer Immune Monitoring and Analysis Centers (CIMAC) to standardize and validate a mIF workflow [38]. A third source of error was heterogeneity of sTIL counts across areas of the tumor. One proposed solution to mitigate this error is to sample and average sTIL counts across multiple ROIs [13]. Using mIF, it is feasible to estimate sTIL counts across a large number of ROIs, and we demonstrate that adequate ROI sampling is important for stabilizing estimates of treatment-related changes in sTILs in the context of clinical trials.

Statistical modeling has been underexplored as a method for improving accuracy and precision of sTIL/IC density estimation. To date, there is no universally adopted approach for the statistical treatment of mIF output data. By convention, many investigators collapse ROI IC density estimates into a mean per-sample score, which does not fully utilize the added information derived from repeated sampling across ROIs. As an alternative, we demonstrate statistical modeling can improve statistical power and minimize potential detrimental confounding effects of intratumoral heterogeneity. As illustrated in Table 3, statistical modeling was associated with a narrowing of confidence intervals of IC estimates, and smaller observed $p$ values. Furthermore, compared to conventional $t$-testing of means, the hierarchical linear modeling method reduced the required patient enrollment size from $n=25$ to $n=13$ to show an effect of IRX-2 on sTILs.

We also illustrate how mIF can be also used to evaluate more complex hypotheses related to I-O treatment effect. For example, based upon preclinical models and previous trials data, it was hypothesized that locoregional cytokine perfusion (IRX-2) would increase lymphocyte trafficking and facilitate PD-L1 upregulation within the breast tumor via modulation of the JAK-STAT pathway [17]. Using mIF, we confirmed that IRX-2 is associated with increases in sTILs and PD-L1 upregulation in the tumor microenvironment, as well as a shift in the ratio of cytotoxic $\mathrm{T}$ cells to CD163+ macrophages and regulatory $\mathrm{T}$ cells. These findings are corroborated by previously published data from gene expression profiling, clinical SP142/H\&E sTIL assays, and T cell receptor DNA sequencing [14]. Based upon these encouraging findings, we are conducting a trial to compare singledose anti-PD-1 +/- IRX-2 ( $n=15$ per arm) as an induction therapy to potentially enhance immune infiltration prior to neoadjuvant chemotherapy plus pembrolizumab in stage II-III TNBC (NCT04373031). In the future, the spatial output data derived from mIF can be used to evaluate spatial hypotheses, such as whether cytokine therapy permits aggregation or penetration of tumors into the tumor/stromal interface. Such a hypothesis could be evaluated by comparing pre versus posttreatment densities of buffer zones surrounding the tumor/stromal interface.

Our approach is not without limitations. First, because the assay is limited to 7 markers, B cell 
markers were not included, and this may have influenced the overall estimation of sTILs (since B cells would be included in the H\&E sTIL score). It is possible to customize mIF with different markers; however, careful attention must be paid to ensure that each panel is properly validated using ESBC specimens, and therefore for this pilot study, we opted to use a previously validated panel for which we have extensive experience and publication [21, 39]. Future improvements in technology are anticipated to allow for simultaneous measurement of additional markers. A second limitation is the lack of a treatment control, which precluded assessment of potential confounding effects of time and/or biopsy trauma. This will be addressed in the ongoing randomized phase II trial. A third limitation is the resource-intensive nature of our approach, which requires acquisition and analysis of all lymphocyte-bearing ROIs in a given sample. This process may require $24 \mathrm{~h}$ of processing or greater per specimen; however, we illustrate that the efforts are worthwhile in the context of clinical research as they may reduce sample size requirements. In clinical trials, per-patient expenses, time, and effort are likely to far outweigh the added time and cost required to sample more ROIs. Future advances in technology may permit more rapid acquisition and analysis of whole-slide data, for example using the PerkinElmer Polaris system, which is being validated by our group and others. The fourth limitation is that breast cancer subtypes and/or clinical settings may have unique histologic and immunologic features and therefore our power calculations may not be externally valid in other settings. For example, baseline sTIL levels and PD-L1 expression are lower in hormone-sensitive breast cancers relative to TNBC, and therefore when designing a clinical trial, the power analyses would have to be repeated or modified to account for these expected differences in baseline.

As a future direction, the described statistical modeling can be amended to incorporate data on spatial locations of each ROI and/or each cell, which has further potential to improve estimation. For example, because IC densities of immediately adjacent ROIs may be correlated, the accuracy of the model could be improved after adjustment for spatial autocorrelation. Similarly, topographical features such as leading invasive margin of the tumor are expected to influence IC densities and may be accounted for in the model [13]. We are piloting advanced spatial modeling that would enable adjustment of IC densities according to spatial distance from observed topographical landmarks, as well as more advanced methods to exclude non-interpretable areas within ROI to improve accuracy.

\section{Conclusion}

mIF may be used in the context of ESBC I-O trials to detect dynamic changes in ICs and PD-L1 expression associated with treatment. Our mIF method is concordant with H\&E sTIL and SP142 PD-L1 clinical assays, but enhances precision of these measurements by accounting for intratumoral heterogeneity. The statistical modeling approach could also be evaluated as a method of improving performance of the H\&E sTIL clinical assay. The method may also be used to evaluate other features of the immune response, such as treatment-related changes in cellular ratios, or immune cell clustering patterns. Our approach requires validation, which is planned using specimens from a phase II neoadjuvant phase II trial of pembrolizumab +/- IRX-2. (NCT04373031). The method also has promise to be explored as a predictive biomarker in the context of neoadjuvant anti-PD-1/L1 plus chemotherapy, as PD-L1 expression alone did not predict benefit in this setting [40].

\section{Supplementary Information}

The online version contains supplementary material available at https://doi. org/10.1186/s13058-020-01378-4.

\footnotetext{
Additional file 1: Table S1. Estimated cell count ratios. Table S2. Power analysis using Monte Carlo simulation approach ( $n=1000$ simulations), based on the generalized linear mixed-effects model and the observed data structure [i.e., effect sizes and variations obtained post - hoc from a pilot experiment]. Table S3. PD-L1 and STIL scores for individual patients with coefficients of variation.
}

Additional file 2: Figure S1. Receiver operating characteristic curve for determining PD-L1 threshold. (A) Example images of high powered (20x) ROIs, InForm pathology view (showing only PD-L1 expression by $\mathrm{mIF}$ ) counterstained with DAPI. Green is used here to label random cells that could be visually classified as PD-L1 positive versus negative by the reading pathologist, and used to ascertain an appropriate QIF cutoff for PD-L1 positivity. (B) Histogram of the distribution average per-cell QIF PD-L1 levels of 55,108 cells pooled from $24 \mathrm{ROI}$ across 4 patients). (C) ROC curve illustrating sensitivity and specificity for given PD-L1 QIF thresholds. A threshold of $\geq 2.6$ was selected, corresponding with sensitivity of $91 \%$, specificity of $99 \%$, AUC of 0.97 , and accuracy of $95 \%$. ROl: region of interest; PD-L1: programmed death ligand 1; mIF: multispectral immunofluorescence; DAPI: 4',6-diamidino-2-phenylindole; QIF: quantitative immunofluorescence; ROC: receiver operating characteristic; AUC: area under the curve.

Additional file 3: Figure S2. Example of mIF staining and illustration of QIF levels across phenotypes using machine-learning based (InForm) phenotyping method. PD-L1: programmed death ligand 1; DAPI: 4',6-diamidino-2-phenylindole; CK: cytokeratin; FOXP3. (A) mIF images showing CK+ tumor nests with CD8 expression (red), CD3 expression (green), and yellow indicating co-expression; (B) expected expression patterns of helper T cells, cytotoxic T cells, and regulatory T cells; (C-D) Mean quantitative immunofluorescence of CD3 and CD8 for each of the phenotypes; (E-G) Comparison of mean QIF expression patterns for various phenotypes macrophage CD163 expression to other cells. CK: cytokeratin; FOXP3: forkhead box P3; reg: regulatory; QIF: quantitative immunofluorescence.

Additional file 4: Figure S3. mIF antibody validation. Antibody validation was performed using conventional/standard chromogenic stain on human FFPE tonsil tissue. TSA-Opal fluorescent stain was performed on the adjacent slides. Images were acquired with a Vectra 3 Automated Quantitative Pathology Imaging system. FOXP3: forkhead box P3; CK: cytokeratin; PD-L1: programmed death ligand 1. 
Additional file 5: Figure S4. $\mathrm{mIF}$ antibody validation, PD-L1 staining. Antibody validation (aPD-L1; clone E1L3N) was performed using conventional/standard chromogenic stain on human FFPE placenta tissue. TSAOpal fluorescent stain was performed on the adjacent slides. Images were taken with a Vectra 3 Automated Quantitative Pathology Imaging system. PD-L1: programmed death ligand 1; H\&E: Hematoxylin and eosin.

\section{Abbreviations}

AUC: Area under the curve; Cl: Confidence interval; CK: Cytokeratin; CV: Coefficient of variation; ESBC: Early-stage breast cancer; FC: Fold change; FDA: Food and Drug Association; FFPE: Formalin fixed paraffin embedded; GLMM: Generalized linear mixed model; H\&E: Hematoxylin and eosin; IO: Immuno-oncology; IC: Immune cell; IRB: Institutional review board; MDSC: Myeloid-derived suppressor cell; mIF: Multiplex immunofluorescence; PD-L1: Programmed death ligand 1; QIF: Quantitative immunofluorescence; ROI: Region of interest; STLL: Stromal tumor-infiltrating lymphocyte; TC: Tumor cell; TNBC: Triple negative breast cancer

\section{.Acknowledgements}

We acknowledge the following for contributing to patient enrollment: Kelly S. Perlewitz, MD, Janet Ruzich, MD, Alison Conlin, MD, Anupama Acheson, MD, Kristen Massimino, MD, Shaghayegh Aliabadi-Wahle, MD, and James Imatani, MD. We acknowledge Nicole Moxon, RN, and Staci Mellinger, RN, and Tracy Kelly for caring for patients on this trial. We acknowledge the Brooklyn ImmunoTherapeutics team (Lynn Sadowski-Mason, Monil Shah, and others) for their ongoing support and contributions to the study.

\section{Authors' contributions}

KS contributed to study concept, study design, data acquisition, data analysis, data interpretation, software development, and manuscript drafting. KS also contributed to the imaging and analyses of output data. IK contributed to data acquisition and data analysis. BC contributed to data acquisition and data analysis. JP contributed to data acquisition and data analysis. WR contributed to study concept, study design, data interpretation, and manuscript drafting. WU contributed to study concept and manuscript drafting. MM contributed to data acquisition, data analysis, and data interpretation and was one of two study pathologists who evaluated specimens and conducted sTIL H\&E scoring. YW contributed to data acquisition, data analysis, and data interpretation and was one of two study pathologists who evaluated specimens and conducted sTIL H\&E scoring. MC contributed to the data acquisition. ZS contributed to study design, data acquisition, and data analysis. ZH also conducted the histology staining and contributed to the imaging and analysis of output data. GG contributed to study design, data analysis, and data interpretation. SC contributed to study design, data analysis, data interpretation, software development, and manuscript drafting and serves as the study biostatistician. BB contributed to study design, data analysis, data interpretation, and software development. DP contributed to study concept, study design, data acquisition, data analysis, data interpretation, and manuscript drafting. He is the PI of the study and oversaw the entirety of the project. The author(s) read and approved the final manuscript.

\section{Authors' information}

Not applicable.

\section{Funding}

Funding for the clinical trial and data collection was provided by Brooklyn Immunotherapeutics. Support was provided by Frank \& Mary Gill (Portland, OR) and Walter Bowen (Portland, OR) via the Providence Portland Medical Foundation.

\section{Availability of data and materials}

The full mIF datasets will be furnished upon request at a later date after upon completion of remaining analyses and publication.

\section{Ethics approval and consent to participate}

The clinical trial was approved and overseen by the Providence Health \& Services Institutional Review Board. Informed consent was obtained per institutional guidelines for each subject.

\section{Consent for publication}

Not applicable.

\section{Competing interests}

KS has no competing interests to declare. IK has no competing interests to declare. $\mathrm{BC}$ has no competing interests to declare. JP has no competing interests to declare. WR has received research funding from Bristol-Myers Squibb, Merck, Nektar Therapeutics, GSK, Galectin Therapeutics, Inhibrx, Oncosec, Aeglea Biotherapeutics, Veana Therapeutics, and MiNA Therapeutics. WR has also received advisory board honoraria from Nektar Therapeutics and serves on the advisory board of Vesselon, Inc.

WU has no competing interests to declare. MM has no competing interests to declare. YW has no competing interests to declare. MC has no competing interests to declare. ZS has no competing interests to declare. GG has no competing interests to declare. SC has no competing interests to declare. $\mathrm{BB}$ has no competing interests to declare.

DP has received advisory board honoraria and institutional research funding support from Brooklyn ImmunoTherapeutics, Bristol-Myers Squibb, and Merck Laboratories. DP receives speaker bureau honoraria from Genentech and Novartis. Unrelated to this work, DP has received additional advisory board honoraria from other entities.

\section{Author details}

${ }^{1}$ Earle A. Chiles Research Institute, 4805 N.E. Glisan St., North Tower, Suite 2N87, Portland, OR 97213, USA. 'Providence Cancer Institute, Portland, OR, USA. ${ }^{3}$ Department of Pathology, Providence Portland Medical Center, Portland, OR, USA. ${ }^{4}$ Medical Data Research Center, Providence Health \& Services, Portland, OR, USA.

Received: 27 April 2020 Accepted: 3 December 2020

Published online: 07 January 2021

\section{References}

1. Schmid P, Adams S, Rugo HS, Schneeweiss A, Barrios $\mathrm{CH}$, Iwata H, et al. Atezolizumab and nab-paclitaxel in advanced triple-negative breast cancer. N Engl J Med. 2018;379(22):2108-21.

2. Schmid P, Adams S, Rugo HS, Schneeweiss A, Barrios $\mathrm{CH}$, Iwata H, et al. IMpassion130: updated overall survival (OS) from a global, randomized, double-blind, placebo-controlled, Phase III study of atezolizumab (atezo) + nab-paclitaxel (nP) in previously untreated locally advanced or metastatic triple-negative breast cancer (mTNBC). J Clin Oncol. 2019;37(15_suppl):1003.

3. Page DB, Bear H, Prabhakaran S, Gatti-Mays ME, Thomas A, Cobain E, et al. Two may be better than one: PD-1/PD-L1 blockade combination approaches in metastatic breast cancer. NPJ breast cancer. 2019;5:34.

4. Gatti-Mays ME, Balko JM, Gameiro SR, Bear HD, Prabhakaran S, Fukui J, et al. If we build it they will come: targeting the immune response to breast cancer. NPJ breast cancer. 2019;5:37.

5. Adams S, Gatti-Mays ME, Kalinsky K, Korde LA, Sharon E, Amiri-Kordestani L, et al. Current landscape of immunotherapy in breast cancer: a review. JAMA Oncol. 2019;5(8):1205-14

6. Vennapusa B, Baker B, Kowanetz M, Boone J, Menzl I, Bruey JM, et al. Development of a PD-L1 complementary diagnostic immunohistochemistry assay (SP142) for atezolizumab. Appl Immunohistochem Mol Morphol. 2019; 27(2):92-100.

7. Salgado R, Denkert C, Demaria S, Sirtaine N, Klauschen F, Pruneri G, et al. The evaluation of tumor-infiltrating lymphocytes (TILS) in breast cancer: recommendations by an International TILs Working Group 2014. Ann Oncol. 2015;26(2):259-71.

8. Loi S, Drubay D, Adams S, Pruneri G, Francis PA, Lacroix-Triki M, et al. Tumor-infiltrating lymphocytes and prognosis: a pooled individual patient analysis of early-stage triple-negative breast cancers. J Clin Oncol. 2019; 37(7):559-69.

9. Denkert C, von Minckwitz G, Darb-Esfahani S, Lederer B, Heppner BI, Weber $\mathrm{KE}$, et al. Tumour-infiltrating lymphocytes and prognosis in different subtypes of breast cancer: a pooled analysis of 3771 patients treated with neoadjuvant therapy. Lancet Oncol. 2018;19(1):40-50. 
10. Stanton SE, Disis ML. Clinical significance of tumor-infiltrating lymphocytes in breast cancer. J Immunother Cancer. 2016;4:59.

11. Voorwerk L, Slagter M, Horlings HM, Sikorska K, van de Vijver KK, de Maaker $M$, et al. Immune induction strategies in metastatic triple-negative breast cancer to enhance the sensitivity to PD-1 blockade: the TONIC trial. Nat Med. 2019;25(6):920-8.

12. Loi S, Winer E, Lipatov O, Im S-A, Goncalves A, Cortes J, et al. Abstract PD503: Relationship between tumor-infiltrating lymphocytes (TILS) and outcomes in the KEYNOTE-119 study of pembrolizumab vs chemotherapy for previously treated metastatic triple-negative breast cancer (mTNBC). Cancer Res. 2020;80(4 Supplement):PD5-03-PD5.

13. Kos Z, Roblin E, Kim RS, Michiels S, Gallas BD, Chen W, et al. Pitfalls in assessing stromal tumor infiltrating lymphocytes (sTILs) in breast cancer. NPJ Breast Cancer. 2020;6:17.

14. Page DB, Pucilowska J, Sanchez KG, Conlin AK, Acheson AK, Perlewitz KS, et al. A phase Ib study of pre-operative, locoregional IRX-2 cytokine immunotherapy to prime immune responses in patients with early stage breast cancer. Clin Cancer Res. 2020;26(7):1595-605.

15. Berinstein NL, Wolf GT, Naylor PH, Baltzer L, Egan JE, Brandwein HJ, et al, Increased lymphocyte infiltration in patients with head and neck cancer treated with the IRX-2 immunotherapy regimen. Cancer Immunol Immunother. 2012;61(6):771-82.

16. Wolf GT, Fee WE Jr, Dolan RW, Moyer JS, Kaplan MJ, Spring PM, et al. Novel neoadjuvant immunotherapy regimen safety and survival in head and neck squamous cell cancer. Head Neck. 2011;33(12):1666-74.

17. Berinstein NL, McNamara M, Nguyen A, Egan J, Wolf GT. Increased immune infiltration and chemokine receptor expression in head and neck epithelial tumors after neoadjuvant immunotherapy with the IRX-2 regimen. Oncoimmunology. 2018;7(5):e1423173.

18. Czystowska M, Szczepanski MJ, Szajnik M, Quadrini K, Brandwein H, Hadden $\mathrm{JW}$, et al. Mechanisms of T-cell protection from death by IRX-2: a new immunotherapeutic. Cancer Immunol Immunother. 2011;60(4):495-506.

19. TILs in breast cancer 2020 [Available from: https://www.tilsinbreastcancer. org/. Accessed 15 Dec 2020.

20. Ventana PD-L1 (SP142) Assay Interpretation Guide 2019 [Available from: https://diagnostics.roche.com/content/dam/diagnostics/us/en/resourcecenter/NENTANA-PD-L1-(SP142)-Assay-Interpretation-Guide.pdf. Accessed 15 Dec 2020.

21. Baird JR, Bell RB, Troesch V, Friedman D, Bambina S, Kramer G, et al. Evaluation of explant responses to STING ligands: personalized immunosurgical therapy for head and neck squamous cell carcinoma. Cancer Res. 2018:78(21):6308-19.

22. Hodgson A, Slodkowska E, Jungbluth A, Liu SK, Vesprini D, Enepekides D, et al. PD-L1 immunohistochemistry assay concordance in urothelial carcinoma of the bladder and hypopharyngeal squamous cell carcinoma. Am J Surg Pathol. 2018;42(8):1059-66.

23. Stack EC, Wang C, Roman KA, Hoyt CC. Multiplexed immunohistochemistry, imaging, and quantitation: a review, with an assessment of Tyramide signal amplification, multispectral imaging and multiplex analysis. Methods. 2014; 70(1):46-58

24. inForm User Manual Version 2.4.2 2019 [Available from: https://research. pathology.wisc.edu/wp-content/uploads/sites/510/2018/12/ inFormUserManual_2_4_2_rev0.pdf. Accessed 15 Dec 2020.

25. De Angelis C, Nagi C, Hoyt CC, Liu L, Roman K, Wang C, et al. Evaluation of the predictive role of tumor immune infiltrate in patients with HER2positive breast cancer treated with neoadjuvant anti-HER2 therapy without chemotherapy. Clin Cancer Res. 2020;26(3):738-45.

26. Fitzmaurice GM, Laird NM, Ware JH. Applied longitudinal analysis. 2nd ed. Hoboken: Wiley; 2011.

27. McCullagh P, Nelder JA. Generalized Linear Models. 2nd ed. London: Chapman and Hall; 1989

28. Brown JR, Bai Y, Bossuyt V, Nixon C, Lannin DR, Rimm DL. Quantitative assessment of CD3, CD8, and CD20 in tumor-infiltrating lymphocytes and predictive value for response to neoadjuvant chemotherapy in breast cancer. ASCO Meeting Abstracts. 2014;32(15_suppl):1027.

29. Green P, MacLeod CJ. SIMR: an R package for power analysis of generalized linear mixed models by simulation. Methods Ecol Evol. 2016;7(4):493-8.

30. Van Belle G, Martin DC. Sample size as a function of coefficient of variation and ratio of means. Am Stat. 1993;47(3):165-7.
31. Stagg J, Loi S, Divisekera U, Ngiow SF, Duret H, Yagita H, et al. Anti-ErbB-2 $\mathrm{mAb}$ therapy requires type I and II interferons and synergizes with anti-PD-1 or anti-CD137 mAb therapy. Proc Natl Acad Sci U S A. 2011;108(17):7142-7.

32. Linch SN, Kasiewicz MJ, McNamara MJ, Hilgart-Martiszus IF, Farhad M, Redmond WL. Combination OX40 agonism/CTLA-4 blockade with HER2 vaccination reverses T-cell anergy and promotes survival in tumor-bearing mice. Proc Natl Acad Sci U S A. 2016;113(3):E319-27.

33. Messenheimer DJ, Jensen SM, Afentoulis ME, Wegmann KW, Feng Z, Friedman DJ, et al. Timing of PD-1 blockade is critical to effective combination immunotherapy with anti-OX40. Clin Cancer Res. 2017;23(20): 6165-77.

34. Cortazar P, Zhang L, Untch M, Mehta K, Costantino JP, Wolmark N, et al. Pathological complete response and long-term clinical benefit in breast cancer: the CTNeoBC pooled analysis. Lancet. 2014;384(9938):164-72.

35. Yeong J, Tan T, Chow ZL, Cheng Q, Lee B, Seet A, et al. Multiplex immunohistochemistry/immunofluorescence (mIHC/IF) for PD-L1 testing in triple-negative breast cancer: a translational assay compared with conventional IHC. J Clin Pathol. 2020;73(9):557-62.

36. Lu S, Stein JE, Rimm DL, Wang DW, Bell JM, Johnson DB, et al. Comparison of biomarker modalities for predicting response to PD-1/PD-L1 checkpoint blockade: a systematic review and meta-analysis. JAMA Oncol. 2019:5(8): 1195-204.

37. Gonzalez-Ericsson PI, Stovgaard ES, Sua LF, Reisenbichler E, Kos Z, Carter JM, et al. The path to a better biomarker: application of a risk management framework for the implementation of PD-L1 and TILs as immuno-oncology biomarkers in breast cancer clinical trials and daily practice. J Pathol. 2020; 250(5):667-84

38. Akturk G, Cuentas ERP, Lako A, Gjini E, Espiridion BS, Wistuba II, et al. CIMACCIDC tissue imaging harmonization. J Clin Oncol. 2020;38(15_suppl):3125.

39. Sanchez K, Kim I, Chang S, Martel M, Yu W, Bernard B, et al. Multispectral immunofluorescence (mIF) to detect dynamic changes in PD-L1 expression, immune cell $(\mathrm{IC})$ infiltration, and tumor-IC interactions in primary breast cancer (BC) immuno-oncology clinical trials. San Antonio: SABCS; 2019.

40. Schmid P, Cortes J, Pusztai L, McArthur H, Kummel S, Bergh J, et al. Pembrolizumab for early triple-negative breast Cancer. N Engl J Med. 2020; 382(9):810-21.

\section{Publisher's Note}

Springer Nature remains neutral with regard to jurisdictional claims in published maps and institutional affiliations.
Ready to submit your research? Choose BMC and benefit from:

- fast, convenient online submission

- thorough peer review by experienced researchers in your field

- rapid publication on acceptance

- support for research data, including large and complex data types

- gold Open Access which fosters wider collaboration and increased citations

- maximum visibility for your research: over $100 \mathrm{M}$ website views per year

At BMC, research is always in progress.

Learn more biomedcentral.com/submissions 